\title{
THE ALINEMENT OF ASTEROID ROTATION
}

\author{
JOSEPH A. BURNS \\ Cornell University
}

Data have been accumulating since the beginning of this century that indicate that most, if not all, large asteroids have periodic lightcurves. The variations that are seen have periods of the order of several hours and can be understood as being caused by bodily rotation, accompanied by changes in shape and/or surface properties. Because corresponding color changes are usually absent, the former explanation of a variation in cross section is probably the correct one.

The lightcurves of the asteroids do not exhibit photometric beat phenomena and, as Kopal (1970) has argued, this indicates that the rotation is about only one axis. In point of fact, the pole of the rotation axis can be determined from observations. ${ }^{1}$ The principle behind interpreting these observations is easily understood: If, for simplicity, we assume that an asteroid orbits in the ecliptic and that its brightness is proportional to the surface area seen, then any variation in brightness (after corrections for distance and phase effects have been made) must correspond to a variation in the projected surface area. There will be no change in the brightness variation over one orbital period if the rotation pole is normal to the orbit plane, for then the differences in surface area seen over one rotation period are the same everywhere on the orbit. On the other hand, the maximum changes in surface area and, hence, the maximum brightness variation will occur when the rotation pole lies in the orbit plane; intermediate variations will correspond to intermediate positions. So, by observing the variation in the magnitude of the brightness over one orbital period, one can evaluate the longitude and latitude of the asteroid's rotation pole. An approximate technique, based on this idea but using only a few observations, has been applied to many asteroids in a series of papers primarily by Kuiper and Gehrels with others. (See Dunlap and Gehrels, 1969; Gehrels, 1967; Gehrels and Owings, 1962; Gehrels et al., 1970; Vesely;2 and Wood and Kuiper, 1962.) The results, which could be further refined, indicate that the rotation axes may be clustered in ecliptic longitude and that almost all asteroids have large obliquities; the only one of the 15 or so whose rotation

\footnotetext{
${ }^{1}$ See p. 127.

${ }^{2}$ See p. 133.
} 
axis lies more than $20^{\circ}$ from the ecliptic is the large and nearly spherical Vesta (Gehrels et al., 1970) whose pole appears to be at about $65^{\circ}$ ecliptic latitude.

The result that the rotation is about only one axis is truly surprising. According to rigid body dynamics, only when a principal axis lies along the direction defined by the body's angular momentum vector $\mathbf{H}$ will there be no precession. Otherwise, the principal axis system $x y z$ fixed to the body should freely precess about $\mathbf{H}$. We define $C>B>A$ to represent the moments of inertia about the $z, y$, and $x$ axes, respectively, and consider as an example the case where the angular velocity $\boldsymbol{\omega}$ lies near the maximum axis $z$ of inertia. Then the precession has an angular velocity $(\alpha \delta)^{1 / 2} \omega_{z}$ where

Furthermore

$$
\alpha=\frac{C-B}{A} \quad \delta=\frac{C-A}{B}
$$

$$
\left(\frac{\omega_{x}}{\omega_{z}}\right)^{2} \sim \alpha \quad\left(\frac{\omega_{y}}{\omega_{z}}\right)^{2} \sim \delta
$$

(Symon, 1960). For typical asteroid shapes, $\alpha$ and $\delta$ range from $10^{2}$ to $10^{-2}$ and thus the free precession will occur on a time scale that is within an order of magnitude or two of the rotation time scale; in other words, it would be observable if it existed and $\boldsymbol{\omega}$ were not closely alined with $\mathbf{H}$.

Dynamics also tells that the rotation will be stable only if it is about $z$, the axis of maximum moment of inertia, or $x$, the minimum axis. Observations are in agreement with this: The asteroids appear to be spinning about the maximum axis. This latter fact indicates that energy dissipation may be taking place because convergence of the maximum axis with $\mathbf{H}$ generally will occur whenever energy is dissipated internally in a quasi-rigid body (Pringle, 1966).

Kopal (1970) has argued that the absence of any precession indicates that the asteroids could not have arisen from collisions because then one should expect a random distribution of their angular momenta with respect to their body axes; thus he believes asteroids must have been formed in their present alined state. We wish to present a different interpretation of the peculiar alinement phenomenon.

We will present directly below calculations showing that at least a few major impacts should have taken place on the large asteroids after their formation. Such collisions will change each asteroid's $\mathbf{H}$ and will, in general, misaline $\mathbf{H}$ from $\boldsymbol{\omega}$. Thus, even if the rotation axes were perfectly alined originally, precession of some asteroids should be observed today. Because it is not seen, an alining mechanism must be (or must have been) at work if the collision calculations are correct. This idea receives some further support from the unusual ordering of the orientations of the rotation axes that itself speaks of an alinement process; it is quite difficult to explain Gehrels' large obliquities and the clustering in ecliptic longitude without some such process.

Before discussing possible alining mechanisms, let us first consider the collision process. Collisions between at least small asteroids are generally 
believed to be still occurring; they are frequently invoked as a mechanism to provide material for the zodiacal dust cloud and for some meteorites. To find the number $n$ of asteroids that are large enough to measurably affect the angular momenta of the visible asteroids, we recall that a collision between two bodies of masses $m$ and $M$ will change the angular momentum of the $M$ body on the order of $R m v$, where $R$ is a mean radius and $v$ is the impact velocity. The impact does not change the initial orientation of the asteroid but does instantaneously affect $\boldsymbol{\omega}$ and $\mathbf{H} ; \mathbf{H}$ swings in space through an angle of the order of $m v / M \omega R$ radians. Taking an average relative velocity of $5 \mathrm{~km} / \mathrm{s}$, about one-third the Kepler orbit speed (Wetherill, 1967), $R=40 \mathrm{~km}$ and $2 \pi / \omega=5 \mathrm{hr}$, collisions with bodies of $m / M>3 \times 10^{-4}$ will cause $\mathbf{H}$ to rotate on the average by more than $5^{\circ}$. These collisions should produce a perceptible precession; for $R=40 \mathrm{~km}$ there will be $10^{4}$ to $10^{5}$ particles capable of producing this precession (Allen, 1963).

An asteroid's mean collision time scale $\tau$ can be approximated by a particle-in-a-box calculation: We consider that all the asteroids move within a torus of elliptical cross section whose volume $V$ is approximately

$$
2 \pi \bar{a} \pi(\bar{a} \sin \bar{i})(2 \bar{a}) \quad \text { or } \sim 5 \times 10^{40} \mathrm{~cm}^{3}
$$

where $\bar{a}, \bar{e}$, and $\bar{i}$ are, respectively, the mean semimajor axis, the mean eccentricity, and the mean inclination of the visible asteroids (Allen, 1963). Now $\tau$ is found by dividing the torus volume by the number of possible colliding particles multiplied by the collision cross section times the average velocity difference between two asteroids; i.e., the $v$ from above. So

$$
\tau=\frac{V}{\nu n \pi R^{2}}
$$

or $10^{8}$ to $10^{9} \mathrm{yr}$. The results of more detailed work (Anders, 1965; Hartmann and Hartmann, 1968; Wetherill, 1967) agree quite well with this rough calculation. If present densities have existed throughout the past, most asteroids having a mean radius $R=\mathbf{4 0} \mathrm{km}$ will have been struck many times during their lifetimes by particles massive enough to change their $\mathbf{H}$ by at least $5^{\circ}$. Larger particles will be so affected less frequently; $\tau$ for a $100 \mathrm{~km}$ body is just about the age of the solar system. Thus it is more likely that a medium-size asteroid should be seen precessing than a very large one. Naturally the current photometric data are primarily of the larger asteroids with many having radii about $100 \mathrm{~km}$ and only several with $R<50 \mathrm{~km}$. The arguments presented here would be strengthened if data could be obtained on more medium-size asteroids.

We now wish to discuss briefly some factors that may affect the final rotation of an asteroid; namely, the influence of melting, aerodynamic drag, internal damping, and electromagnetic dissipation.

Asteroid melting during the Sun's T Tauri phase, as postulated by the unipolar generator mechanism of Sonett et al. (1970; see Sonett's paper in this 
volume ${ }^{3}$ ), would have a profound effect on the asteroid's rotational properties. In fact, with complete melting there would be perfect alinement along the major principal axis; this complete melting would, however, symmetrize the body and this is not seen today. The effects of partial internal melting are difficult to discern at this time but they should produce an immediate partial alinement and accelerate any damping mechanisms. However, this melting would occur early in the evolution of the solar system (if at all) and thus, many of the misalining collisions postulated above will take place subsequently. Hence the alinement seen today apparently cannot be ascribed to a melting that occurred eons ago.

Dissipative aerodynamic torques have been shown to sometimes have a stabilizing effect on the rotation of satellites. Johnson (1968), using many simplifying assumptions and a complicated analysis, has given a stability criterion for cylindrical satellites in terms of a ratio of moments of inertia and body dimensions; applying this to uniform density bodies shows that they always tend to aline themselves along the minimum axis in the presence of aerodynamic torques. The decay time is very long, even in Earth's atmosphere. Although we might expect similar effects due to dust interactions to occur on asteroids, they should be very small; however, Johnson's idealized analysis leaves much to be desired and the problem needs to be studied further.

Let us now discuss internal damping mechanisms. Recently Kopal (1970) has dealt with the damping arising from the most obvious force, gravity. We consider the same problem in a somewhat different manner. The period of the forced precession of an axially symmetric asteroid due to the gravitational torque exerted by a disturbing body of mass $\mu$ is

$$
p=\frac{4 \pi r^{3} C \omega}{3 G \mu(C-A) \cos \epsilon}
$$

where $r$ is the distance between the bodies, $\epsilon$ is the asteroid's obliquity, and $G$ is the universal gravitational constant (Kaula, 1968). If the disturbing body is the Sun, this is of the order of $\omega /\left(n^{2} J_{2} \cos \epsilon\right)$ where $J_{2}=(A-C) / M R^{2}$ and $n$ is the asteroid's orbital mean motion. Using reasonable values of the variables, $P$ is $10^{7}$ or $10^{8} \mathrm{yr}-$ far too long to be observed. One can use equation (2) to find a period of similar magnitude for the precession caused by Jupiter. Because the rate of damping of the precessional motion should occur with a time scale of at least an order or two greater than $P$, we find, in agreement with Kopal (1970), that Jovian-solar effects most likely cannot account for the alinement.

Prendergast (1958) in a brief conference report has summarized unpublished calculations on the internal damping of energy in a mechanism that is driven by the free precessional motion; this work was pointed out to me at this colloquium by G. P. Kuiper. Prendergast's persuasive physical arguments and his results will be repeated here. In a freely precessing body each element that

\footnotetext{
${ }^{3}$ See p. 239.
} 
lies off the instantaneous rotation axis will have an elastic strain as a result of the instantaneous centrifugal acceleration. The elastic strain energy stored by any element in a freely precessing body will change as the instantaneous rotation axis moves through the body. The total strain energy will decrease because each element is in a varying stress field and, thus, loses energy by internal damping. The lost energy ultimately comes from the rotational kinetic energy. As mentioned above, the body accommodates this loss by alining its major principal axis with $\mathbf{H}$ so as to minimize its energy while conserving angular momentum. At this last stage, the axis of rotation is then fixed in the body so that the strains are constant in time and dissipation by this mechanism ceases. The decay times found by Prendergast are of the order of $10^{5} \mathrm{yr}$. This appears to be the alining mechanism we seek; however, unless the calculations themselves become available, one must withhold absolute judgment.

We now ask, is there a dynamical reason that accounts for the observational indication, according to Gehrels' work, that a possible alinement of the rotation axes lies near the ecliptic? (We will ignore Vesta because its free precession time will be very long as a result of its sphericity and any precession it may have will not be observable.) Because the ecliptic is, in some sense, defined by the presence of a planar interplanetary magnetic field, one might seek a mechanism that involves electromagnetic dissipation of energy. Davis and Greenstein (1951) have proposed such a mechanism, using paramagnetic absorption, to explain the polarization of starlight by alining the rotation axes of elongated dust grains with the interstellar magnetic field. This mechanism, when applied to an orbiting body, will cause alinement with the plane of the magnetic field $B$. The time scale over which this phenomenon takes place is $0.1 \chi^{\prime \prime} B^{2} / \omega R^{2}$, where $\chi^{\prime \prime}$ is the imaginary part of the complex susceptibility of the asteroid. Unhappily, this is many orders of magnitude too large to explain the alinement with the ecliptic.

In conclusion, we would like to review briefly the arguments that have been presented. It has been shown that most visible asteroids have suffered at least one major collision in their lifetime and that this collision should have caused subsequent free precession of the asteroid. Because such precession is not observed, mechanisms were sought that would produce alinement. Internal damping, as proposed by Prendergast (1958), seems to account for the body's alinement with the rotation axis. Although the search for an ecliptic alinement mechanism has been unsuccessful, such an alinement mechanism must exist (particularly for the small asteroids) and must have a time scale that is short in comparison with the age of the solar system. The presence of an alining process means that one cannot infer the primordial asteroid rotations from observations made today.

\section{ACKNOWLEDGMENTS}

I thank Dr. J. Veverka for assistance with all aspects of this presentation and D. McAdoo for reading a preliminary manuscript. 


\section{REFERENCES}

Allen, C. W. 1963, Astrophysical Quantities, p. 152. Second ed., Athlone Press. London. Anders, E. 1965, Fragmentation History of Asteroids. Icarus 4, 399-409.

Davis, L., Jr., and Greenstein, J. L. 1951, The Polarization of Starlight by Aligned Dust Grains. Astrophys. J. 114, 206-240.

Dunlap, J. L., and Gehrels, T. 1969, Minor Planets III. Lightcurves of a Trojan Asteroid. Astron. J. 74, 796-803.

Gehrels, T. 1967, Minor Planets. I. The Rotation of Vesta. Astron. J. 72, 929-938.

Gehrels, T., and Owings, D. 1962, Photometric Studies of Asteroids. IX. Additional Light Curves. Astrophys. J. 135, 906-924.

Gehrels, T., Roemer, E., Taylor, R. C., and Zellner, B. H. 1970, Minor Planets and Related Objects. IV. Asteroid (1566) Icarus. Astron. J. 75, 186-194.

Hartmann, W. K., and Hartmann, A. C. 1968, Asteroid Collisions and Evolution of Asteroidal Mass Distribution and Meteoritic Flux. Icarus 8, 361-381.

Johnson, K. R. 1968, Effect of Dissipative Aerodynamic Torque on Satellite Rotation. J. Spacecr. Rockets 5, 408-413.

Kaula, W. M. 1968, An Introduction to Planetary Physics, p. 183. John Wiley \& Sons, Inc. New York.

Kopal, Z. 1970, The Axial Rotation of Asteroids. Astrophys. Space Sci. 6, 33-35.

Prendergast, K. H. 1958, The Effects of Imperfect Elasticity in Problems of Celestial Mechanics. Astron. J, 63, 412-415.

Pringle, R., Jr. 1966, On the Stability of a Body With Connected Moving Parts. AIAA J. 4, 1395-1404.

Sonett, C. P., Colburn, D. S., Schwartz, K., and Keil, K. 1970, The Melting of Asteroidal-Sized Bodies by Unipolar Dynamo Induction From a Primordial T Tauri Sun. Astrophys. Space Sci. 7, 446-488.

Symon, K. R. 1960, Mechanics, p. 454. Second ed., Addison-Wesley Pub. Co., Inc. Reading, Mass.

Wetherill, G. W. 1967, Collisions in the Asteroid Belt. J. Geophys. Res. 72, 2429-2444.

Wood, H. J., and Kuiper, G. P. 1962, Photometric Studies of Asteroids. X. Astrophys. J. $137,1279-1285$.

\section{DISCUSSION}

DOHNANYI: It seems to me that the influence of impacts on the rotation rate and axis of an asteroid is sensitive to the particular failure mode of the asteroid during such inelastic collisions. If an asteroid is hit by an object large enough to cause a catastrophic collision, a spherical shell of debris, concentric with the (spherical) target asteroid will most likely be ejected from it. There may then be an opportunity for momentum multiplication during such a process with corresponding implications on the realinement of the spin axis of the surviving core.

BURNS: The mass loss and angular momentum change resulting from a catastrophic collision-or, for that matter, from any hypervelocity impact-are difficult to predict. Certainly these quantities will depend strongly upon the particular mode of failure that occurs; i.e., on how much matter is ejected following a collision and how that matter is ejected.

However, the important point, insofar as this presentation is concerned, is that many collisions with relatively small bodies will appreciably misaline the angular momentum vector from the body's spin axis, causing noticeable precession. This misalinement will occur also in the remnants of catastrophic collisions. Furthermore, one can expect that the given expression for the change in the angular momentum direction will be of the right order of magnitude as long as the surviving core retains much of the body's original mass. Of course, most collisions are not catastrophic in the sense we are talking about here and in fact the middle-sized collisions should determine how the angular momentum vector changes direction for most bodies. 